\title{
Descripción del patrón y el recorrido de la apertura mandibular en niños con dentición mixta
}

Ramírez $S^{1}$

Espinosa $I^{2}$

Muñoz $G^{2}$

\section{Resumen}

Introducción: El recorrido de la apertura mandibular y las desviaciones que se presentan al abrir la boca, pueden ser un parámetro excelente para detectar de manera oportuna cualquier anomalía a nivel de del complejo temporomandibular.

El recorrido normal de la apertura mandibular en el adulto se encuentra entre los 53 y los 58 $\mathrm{mm}$, con un promedio de $40 \mathrm{~mm}$, un niño a partir de los 7 años puede lograr estos promedios de apertura mandibular. Solo el 1.2\% de los adultos jóvenes abren menos de $40 \mathrm{~mm}$, por lo tanto una apertura mandibular menor puede ser calificada como restringida de acuerdo a la edad y la talla del paciente.

Con el objetivo de determinar el patrón y el recorrido de la apertura mandibular con un método de diagnóstico validado, en un estudio ob- servacional, descriptivo, se incluyeron 139 niños sanos con dentición mixta completa de 8 a 12 años de edad. Los resultados demostraron que el 30.9\% de los niños presentó un patrón de apertura mandibular alterado, siendo la desviación mandibular izquierda corregida la más frecuente $(16.5 \%)$, la apertura mandibularl promedio fue de $50 \pm 4.77 \mathrm{~mm}$, y las lateralidades; derecha $8.3 \pm 3,1 \mathrm{~mm}$, y la izquierda $8.45 \pm 3.00 \mathrm{~mm}$. Lo cual permite concluir que la apertura mandibular en la dentición mixta, se encuentra dentro del recorrido descrito para los adultos en la literatura, con un porcentaje considerable de alteración en el patrón de apertura mandibular, lo cual puede ser considerado uno de los primeros signos de alteración en la función del sistema Temporomandibular.

Palabras Clave: Movimiento, mandíbula, trastornos ATM,dentición mixta,niños

Artigo Original

\section{Descrição do padrão e trajeto de abertura mandibular em crianças com dentição mista}

\section{Resumo}

Introdução: $\mathrm{O}$ trajeto da abertura mandibular e os desvios que ocorrem ao abrir a boca podem ser excelentes parâmetros para detectar de forma oportuna qualquer anomalia no complexo temporomandibular.

\footnotetext{
${ }^{1}$ Odontóloga Pediatra Universidad Autónoma de Puebla, (BUAP), México

${ }^{2}$ Facultad Odontología Universidad Autónoma de Puebla, (BUAP), México
} 
A amplitude normal da abertura mandibular no adulto varia de 53 e $58 \mathrm{~mm}$, com média de 40 $\mathrm{mm}$ sendo que uma criança a partir de 7 anos pode alcançar esses valores. Apenas 1,2\% dos adultos jovens apresentam uma abertura menor que $40 \mathrm{~mm}$. Por conseguinte, considerando a idade e o tamanho do paciente, amplitudes inferiores podem ser descritas como limitadas.

Com o objetivo de determinar o padrão e o trajeto de abertura mandibular com um método de diagnóstico validado; 139 crianças saudáveis com dentição mista completa de 8 a 12 anos de idade foram incluídas em um estudo observacional descritivo. Os resultados indicam 30,9\% das crianças apresentaram um pa- drão alterado de abertura mandibular, sendo o desvio mandibular esquerdo o mais comum $(16,5 \%)$, a média da abertura mandibular média foi de $50 \pm 4,77 \mathrm{~mm}$ e os movimentos laterais; direito $8,3 \pm 3,1 \mathrm{~mm}$ e esquerdo $8,45 \pm 3,00 \mathrm{~mm}$. Assim pode-se concluir que os valores de abertura mandibular na dentição mista encontramse dentro da amplitude descrita, na literatura, para os adultos, com uma porcentagem considerável de alteração no padrão de abertura, que pode ser considerado um dos primeiros sinais de alteração na função do sistema Temporomandibular.

Palavras-chave: Movimentos, mandíbula, dentição mista, desordens ATM, crianças.

\section{Original article}

\section{Patron and path the mandibular opening in children with mixed dentition}

\begin{abstract}
Introduction: The path of the mandibular opening and the mandibular movements' values that show can be an excellent parameter to detect any anomaly timely manner at the level of the temporomandibular complex.

The normal path of the mandibular opening in adults is between 53 and $58 \mathrm{~mm}$, with an average of $40 \mathrm{~mm}$, a child from age 7 can achieve this average mouth opening. Only $1.2 \%$ of young adults open less than $40 \mathrm{~mm}$, therefore a smaller mouth opening can be classified as restricted according to age and size of the patient.
\end{abstract}

The aim of determine the pattern and path of the mandibular opening with a validated diag- nostic method, a descriptive study, included 139 healthy children with mixed dentition full 8 to 12 years old. Results showed that $30.9 \%$ of children presented an altered pattern of mandibular opening, left mandibular deviation being corrected often $(16.5 \%)$, the average mouth opening was $50 \pm 4.77 \mathrm{~mm}$. and lateral movements to the right $8.3 \pm 3.1 \mathrm{~mm}$ and left of $8.45 \pm 3.0 \mathrm{~mm}$. Conclusion: the mandibular opening in the mixed dentition is within the path described for adults in the literature, with a significant rate alteration in the pattern of mouth opening, which can be considered one of the first signs of abnormal function Temporomandibular system.

Key words: movements, TMA disorders, children, mixed dentition 


\section{Introducción}

La apertura mandibular es un movimiento básico que inicia desde la posición dental intercúspidea, seguida por el desplazamiento de la mandíbula hacia abajo y hacia atrás, donde se pierde el contacto entre los dientes superiores y los inferiores. ${ }^{1}$

En la literatura mundial, se reportan valores de los 32 a los 77 milímetros de recorrido de apertura mandibular para la población adulta. Además se ha demostrado que los hombres tienden a abrir en promedio, cinco milímetros más (40 a $77 \mathrm{~mm}$ ) que las mujeres (32 a $75 \mathrm{~mm})^{2,3,4,5}$

La determinación de los patrones del movimiento mandibular y el recorrido de la apertura mandibular son indicadores de la función articular y la musculatura masticatoria, ya que existe una interrelación entre los movimientos mandibulares, el sistema masticatorio y la morfología de la ATM. ${ }^{6}$

De acuerdo con Hansson, los movimientos de la apertura y el cierre mandibular tienen que realizarse sin ninguna desviación lateral de la misma, de lo contrario significa que los cóndilos se mueven asimétricamente y sin coordinación. ${ }^{7}$

Miller y Bookhan consideran que la limitación de la apertura mandibular es un signo precoz e importante de los trastornos temporomandibulares, por lo que desarrollaron un índice en adultos, para determinar la capacidad de la apertura mandibular entre los pacientes con trastornos temporomandibulares de origen miogénico, pacientes con desplazamiento del disco con reducción y grupo control. La media del compromiso de apertura mandibular limitada para los pacientes del grupo miógeno fue de $9.05 \%$, para el grupo de desplazamiento del disco con reducción de $5.7 \%$ y del grupo con- trol de $4.34 \%$, con diferencias estadísticamente significativas $(\mathrm{p}<.001)$ entre el grupo miógeno y el grupo con desplazamiento anterior del disco y $(p<.001)$ entre el miógeno y el grupo control, pero no entre el grupo control y el grupo con desplazamiento anterior del disco. ${ }^{8}$

Varios autores mencionan que el recorrido normal de la apertura mandibular media se encuentra entre los 53 y los 58 milímetros, un niño de 7 años puede abrir un máximo de 51 milímetros. Solo el 1.2\% de los adultos jóvenes abren menos de 40 milímetros, por lo tanto una apertura mandibular menor puede ser calificada como una apertura restringida, de acuerdo a la edad y el tamaño corporal del paciente ${ }^{9,10}$.

Diversos métodos de evaluación han sido utilizados para el registro de la apertura mandibular, por desgracia la alta variabilidad en las formas de medir las aperturas mandibulares máximas normales hacen de la comparación e interpretación de los resultados una tarea difícil. El propósito del presente estudio fue; establecer parámetros objetivos de patrones y recorrido de la apertura mandibular en la dentición mixta en una población mexicana infantil, con un método de diagnóstico validado.

\section{Material y métodos}

Bajo un diseño de estudio: observacional, descriptivo y unicéntrico, se incluyeron 139 niños sanos con dentición mixta de entre 8 y 12 años de edad, de cualquier sexo que asistieron por primera vez a la Clínica de Estomatología Pediátrica de la Facultad de Estomatología de la Benemérita Universidad Autónoma de Puebla. Se excluyeron aquellos niños con uso de aparatología ortodóncica, niños con retraso mental y con diagnóstico de enfermedades sistémicas degenerativas. 
Los pacientes fueron evaluados con los Criterios Diagnósticos para la Investigación de los TTM $(\mathrm{CDI} / \mathrm{TTM})^{9}$, con la participación de una investigadora previamente estandarizada con una correlación de .97 para el registro de la apertura mandibular y kappa de .89 para el registro del patrón de la apertura mandibular.

El recorrido de la apertura mandibular de acuerdo a los CDI/TTM, establece tres medidas: la apertura mandibular confortable (te voy a pedir que abras la boca lo más que puedas sin que sientas ningún tipo de dolor), la apertura máxima no asistida (te voy a pedir que abras la boca lo más que puedas aunque sientas un poco de dolor) y la apertura máxima asistida (te voy a pedir que abras la boca lo más que puedas aunque sientas un poco de dolor, yo con mis dedos voy a ayudarte un poco, cuando esto sea muy molesto me levantas la mano y me detengo), para esta última medida se colocó el pulgar del examinador en el borde de los incisivos superiores y en forma cruzada se colocó el dedo índice sobre los incisivos centrales inferiores y se ejerció una fuerza moderada (110grs) para obtener una palanca y forzar la apertura mandibular. Los niños fueron colocados en el sillón dental en un ángulo de aproximadamente 90 grados, en las tres aperturas se pidió al niño que colocara su mandíbula en una posición cómoda, en todas las mediciones se ubicó el borde de la regla milimetrada en el borde incisal del incisivo central superior que estuviera mejor posicionado verticalmente, se midió la distancia entre éste y el borde incisal del incisivo inferior (distancia interincisal) y se registró la medida (cuando ésta se encontrara entre dos dígitos, se registró siempre el número menor). Una vez obtenidos los tres registros de la apertura mandibular, se sumaron y promediaron y se le sumó o restó la sobremordida vertical de acuerdo al caso. El resultado se de- nominó; apertura mandibular total. Los sujetos que presentaron incisivos centrales en erupción o ausentes, no aplicaron para la medición de las aperturas debido a la dificultad de su registro.

Para el registro del patrón de la apertura mandibular, de acuerdo a los CDI/TTM se colocó el dedo pulgar en el labio inferior del sujeto y se descendió de manera que se facilitara la visualización de los dientes inferiores y se hiciera más evidente la desviación de la línea media. Se pidió al sujeto que abriera la boca lo más que le fuera posible, aún en presencia de dolor, si la desviación mandibular no era clara, se usó una regla milimétrica colocada verticalmente entre las líneas interincisivas superior e inferior como una guía, los patrones de apertura analizados fueron los establecidos de acuerdo a los CDI/ TTM: patrón recto, desviación a la derecha e izquierda corregida y desviación a la derecha e izquierda no corregida u otro tipo de patrón de apertura mandibular. Para el registro de las lateralidades, se solicitó al paciente que desplazara la mandíbula a la derecha tanto como fuera posible a pesar percibir molestia y se registró el desplazamiento de línea interincisal superior a línea interincisal inferior, posteriormente se registró de la misma manera la lateralidad izquierda. A cada una de estas medidas se sumó o restó la desviación (si existiera) de la línea media, según fuera el caso. Finalmente se registró la protrusión solicitándole al paciente que desplazara la mandíbula hacia adelante tanto como fuera posible a pesar de percibir dolor y se registró la distancia de borde incisal superior a borde incisal inferior y se le restó o sumó según fuera el caso, la sobremordida horizontal.

Se realizó análisis estadístico descriptivo para las variables registradas; medidas de tendencia central y de dispersión, el análisis se realizó con el paquete estadístico SPSS versión 17. 


\section{Resultados}

Las características demográficas de la población estudiada se aprecian en el cuadro 1, donde se hace evidente una participación ligeramente superior del sexo masculino y un promedio de edad por arriba de los 9 años.

Cuadro 1. Características demográficas de la población.

\begin{tabular}{|c|c|c|c|c|}
\hline Sexo & Frecuencia & \multicolumn{3}{|c|}{$\%$} \\
\hline Masculino & 73 & \multicolumn{3}{|c|}{52.5} \\
\hline Femenino & 66 & \multicolumn{3}{|c|}{47.5} \\
\hline Total & 139 & \multicolumn{3}{|c|}{100.0} \\
\hline Edad & Mínimo & Máximo & de & IC \\
\hline Media 9.54 & 8 & 12 & 1.217 & 9.36 \\
\hline
\end{tabular}

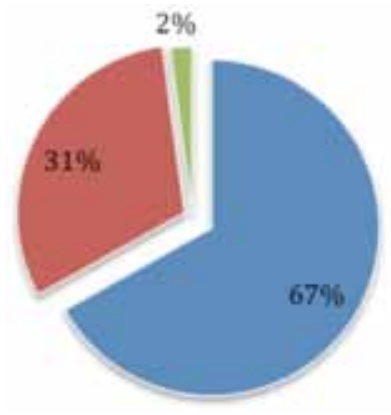

Figura 1. Patrón de apertura mandibular.

" alterado $30.9 \%$

"No aplica $2.2 \%$

Cuadro 3. Recorrido de la apertura mandibular y movimientos mandibulares de la población

\begin{tabular}{|c|c|c|c|c|}
\hline & Mínimo & Máximo & Media & de \\
\hline Apertura confortable & 25 & 55 & 44.59 & 4.726 \\
\hline Apertura máximo no asistida & 26 & 60 & 48.41 & 5.032 \\
\hline Apertura máxima. Asistida & 30 & 65 & 50.15 & 4.984 \\
\hline Apertura promedio & 31 & 62 & 50.35 & 4.777 \\
\hline Lateralidad derecha & 3 & 15 & 8.3 & 3.1 \\
\hline Lateralidad izquierda & 4 & 15 & 8.45 & 3.0 \\
\hline Protrusión & 1 & 19 & 4.8 & 3.4 \\
\hline
\end{tabular}


estándar mínimas y sin mayores restricciones en ninguna de ellas, el desplazamiento mandibular lateral izquierdo fue ligeramente mayor que el derecho, pero ambos dentro de los límites normales y con desviaciones estándar mínimas, la protrusión fue cercana a los $5 \mathrm{~mm}$, como se aprecian en el cuadro 3.

\section{Discusión}

El presente estudio establece que el promedio de la apertura mandibular de los niños con dentición mixta de las clínicas de la Facultad de Estomatología de la BUAP está dentro de los parámetros establecidos en la literatura, sin embargo pone de manifiesto que tres de cada diez niños presentan un patrón de apertura mandibular alterado a pesar de presentar recorridos normales. Una de las fortalezas del presente estudio fue el registro tanto del patrón como el recorrido de la apertura mandibular de acuerdo a lo establecido en un instrumento validado (CDI/TTM). Otra particularidad fue que la población estudiada se encontró en el periodo de la dentición mixta, por lo que se puede establecer dicho parámetro en esta etapa, en comparación con los resultados de otros autores que no consideran a este grupo en particular.

En relación al recorrido de la apertura mandibular máxima encontrada en el presente estudio $(48.41 \mathrm{~mm})$, es similar a la reportada por Ingervall $^{10}$ quien encontró una capacidad de apertura mandibular máxima de $46 \mathrm{~mm}$ en niños de 7 años y ligeramente menor a los $51 \mathrm{~mm}$ en niños de 10 años, con un método de evaluación muy parecido al del presente estudio.

Por otro lado, Yossef-Melis y cols. ${ }^{11}$ encaminaron sus esfuerzos para encontrar un método simple de evaluación de la apertura mandibular máxima, con una herramienta que es proporcional al tamaño del cuerpo, utilizaron el ancho de los tres y cuatro dedos (índice, medio, anular y meñique) en posición horizontal entre los incisivos superiores e inferiores, los cuales posteriormente eran medidos en un vernier y encontraron que los niños de 4 a 15 años presentaron un mínimo de $30 \mathrm{~mm}$, a un máximo de $65 \mathrm{~mm}$, de apertura con una media de $45.8 \mathrm{~mm}$. Esta variación de promedios entre dicho estudio y el presente estudio, pudiera deberse a los rangos de edad y al método utilizado ya que el uso de los dedos entre los incisivos es considerado un método simple y rápido para evaluar la apertura mandibular, sin embargo de exactitud insuficiente y no recomendado para estudios de investigación.

En otros estudios donde se utilizaron los CDI/ TTM, Siriani y cols. reportaron una apertura mandibular total en niños de 6 a 14 años, de $43.70 \mathrm{~mm}$, menor a la reportada en este estudio, los criterios de inclusión de dicho estudio fueron niños sin historia de trauma facial, presencia de todos los incisivos y una buena salud ${ }^{12}$.

Por otro lado, Hirsch-Lautenschläger obtuvieron resultados muy similares en niños y adolescentes alemanes caucásicos de 10 a 17 años, con promedio de 13.1 años, quienes reportaron los movimientos mandibulares y la influencia de la edad, el género, los TTM sobre éstos. Según sus resultados no todos los sujetos que presentan limitaciones en los movimientos mandibulares presentan TTM. Los valores normales de apertura mandibular fueron de $50.6 \mathrm{~mm}$, de 8.2 $\mathrm{mm}$, de protrusión y de $10.2 \mathrm{~mm}$, y $10.6 \mathrm{~mm}$, de lateralidades derecha e izquierda respectivamente ${ }^{13}$, las diferencias en los promedios de los recorridos mandibulares, pudieran deberse a la diferencia de edades.

De acuerdo a diversos autores, la capacidad de apertura mandibular se incrementa de la niñez 
a la edad adulta, sin embargo Sheppard describe que la capacidad de la apertura mandibular es mayor entre los 11 y los 15 años comparada con la capacidad de los adultos. ${ }^{14}$ Finalmente, Cortese y cols. describieron que la apertura mandibular aumenta de la dentición temporal (38.59 $\mathrm{mm}$ ) a la dentición mixta $(41.97 \mathrm{~mm}){ }^{15}$

En relación al patrón de la apertura mandibular, en el presente estudio, el patrón más frecuente fue la desviación mandibular a la izquierda corregida $(16.5 \%)$, en discrepancia con lo reportado por otros autores como Matta ${ }^{16}$, quien registra un patrón de apertura alterado más frecuente en su desviación a la derecha del 9.2\%, mientras que Nilner reporta en el $32 \%$ de su población una desviación mandibular izquierda, sin especificar si hubo o no corrección de dicha apertura y un $1 \%$ de desviación a la derecha.

Otros autores como Grosfeld, Solberg, Vanderas, estudiaron niños y adolescentes en quienes el pa- trón de apertura mandibular con desviación a la izquierda también fue el de mayor prevalencia, sin especificar tampoco si hubo o no corrección ${ }^{17,18}$.

\section{Conclusión}

El presente estudio demostró que la apertura mandibular en niños se encuentra dentro de los recorridos descritos en la literatura, existe un porcentaje elevado del patrón de la apertura mandibular alterado durante este periodo de dentición, el cual puede ser indicador de alteraciones incipientes del complejo temporomandibular, desafortunadamente la amplia variedad de los instrumentos utilizados para evaluar tanto el patrón como el recorrido de la apertura, hacen difícil establecer comparaciones. Es indispensable establecer en la población pediátrica mexicana con dentición mixta la capacidad del recorrido y el patrón de apertura mandibular con instrumentos validados.

\section{Referencias}

1. Posselt. Ulf., Fisiología de la Oclusión y Rehabilitación. Ed. JIM. Barcelona 1973.

2. Zawawi KH, Al-Badawi EA. Lobo Lobo S. Melis M. Melita NR: An index for the measurent of normal maximun mouth opening. J Can Dem Assoc 2003; 69;736-740.

3. Agerberg G: Maximal mandibular movements in young tiieti and women. Swed DemJ 1974; 67:81-100

4. Solberg WK, Woo MW, Houston JB: Prevalence of mandibular dysfunction in young adults. J Am Dent Assoc 1979; 98:25-34.

5. López García Vilma, Gómez Flores, Canseco Jiménez, Evaluación clínica de la disfunción temporomandibular antes del tratamiento ortodóncico. Revista Odontológica Mexicana, Vol. 8, Núm.3, Septiembre 2004, pp 80-89.

6. Moyers, R. Manual de Ortodoncía Clínica. Ed. Panamericana, Buenos Aires, 1992.

7. Hansson T.; et al Dysfuntion Craneomandibular. Editorial Praxis, Barcelona. 1988.

8. Miller V., Bookhan, Brummer, Singh. J.C., A mouth opening index for patients with temporomandibular disorders, Journal of Oral Rehabilitation 1999, 26: 534-537.

9. Dworkin. S. 1992. Approach to the problem. en Research diagnostic crileria for temporomandibular disorders: Review. criteria. examination, and specificalion, critique. Dworkin. S y Le Resche. L (ed,). J. Crancomandib. Disord. Facial Oral Pain. 6:301-355.

10. Invergall Bengt, Range of movement of mandible in children, Scand. J. dent. Res. Department of Orthodontics, Odontological Faculty, University of Gothenburg, Sweden. 1970:78: 311-322. 
11. Youssef S. Abou-Atme, Nada Chedid, Marcello Melis, D.M.D., R.Pharm.; Khalid H. Zawawi, Clinical Measurement of Normal Maximum Mouth Opening in Children, Journal of craniomandibular practice, July 2008, VOL. 26 ,N. 3.

12. Siriani de Oliveira A, Sousa Leticia, Nagamine H, Bevilaqua, Hallak. Evaluation of mandibular range of motion in brazilian chikdren and its correlation to age, height, weight, and gender. Braz Oral Res 2008; 22(1): 61-6.

13. Hirsch C, Lautenschläger C, List T. Mandibular movement capacity in 10-17-yr-old children and adolescents: nosmative values and influence of gender, age, and temporomandibular disorders. Eur J Oral Sci 2006: 114: 465-470.

14. Sheppard. I. M. \&. Sheppard, S. M.: Maximal incisal opening, a diagnostic index J. dent. Med. 1965: 20 : $13-15$.

15. Cortese Silvina, Oliver L, Biondi M. Determination of range of mandibular movements in children without temporomandibular disorders. The Journal Craneomandibular Practice. July 2007, Vol. 25. ํ⒊

16. Matta Carlos, Diez Desilu T. Patrón de desviación de la mandibula durante los movimientos de apertura y cierre bucal según el periodo de dentición en escolares entre 5 y 19 años de edad. La Carta Odontológica, Vol. 5 N¹6, Agosto 2000- Mayo 2001; 31-35.

17. Nilner, M. Prevalence of functional disturbances and discases of stomatognathic system in 7-14 years old. Scand Dent J, 5: 173-187,1981.

18. Grosfeld O; Jackowska M.Czarnecka B. Results of epidemiological examinations of the temporomandibular joint in adolescents and Young adults. J Oral Rehabil, 12:95-105,1985.

Recibido: 26-05-2011

Aceptado: 02-08-2011

Correspondencia: sil_que@hotmail.com 